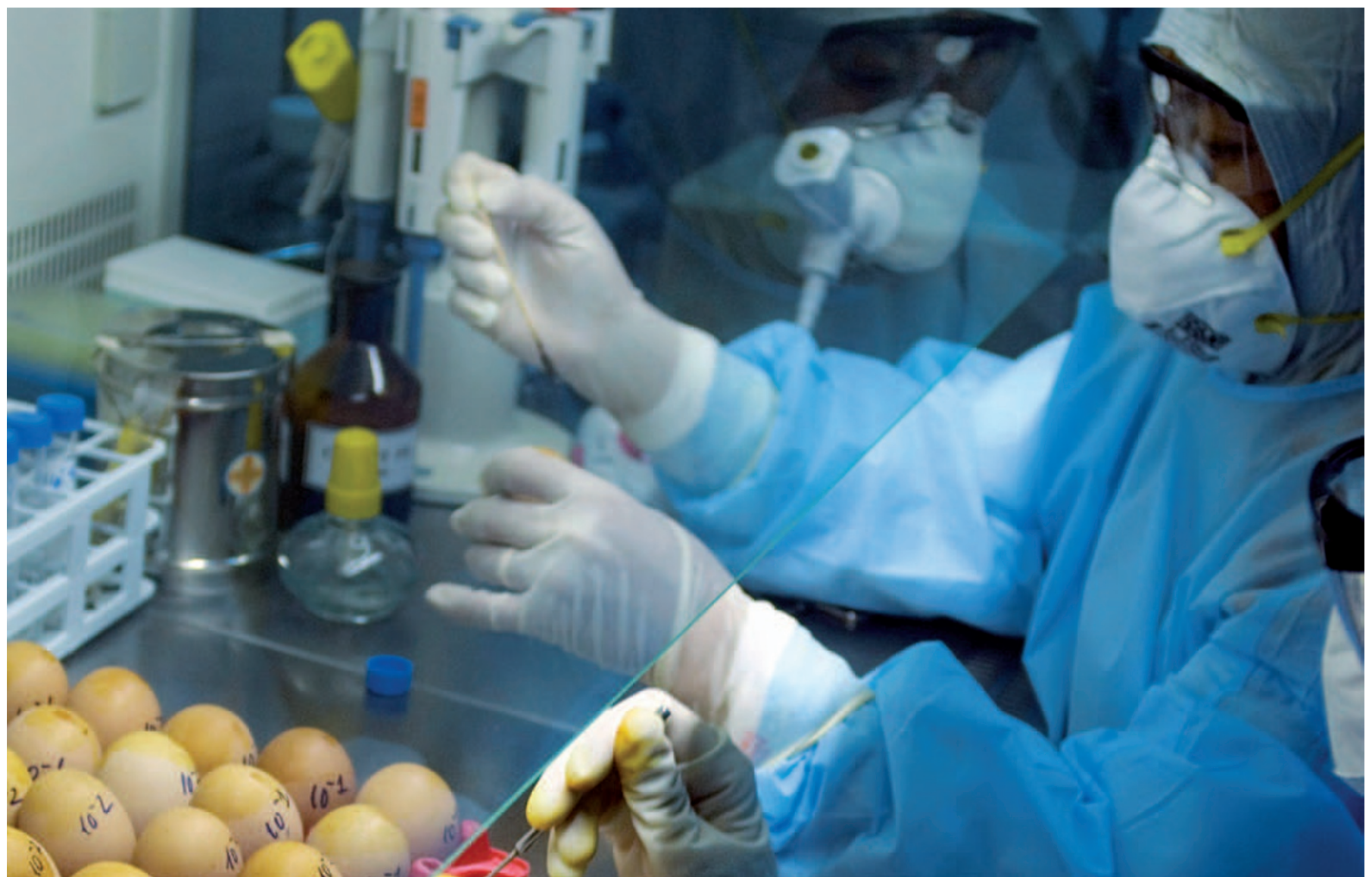

Scientists developing a human vaccine against the avian influenza.

PREVENTION

\title{
Vaccine for all seasons
}

\section{As researchers map the stable parts of the proteins that stud the surface of influenza, the decades-long quest for a universal flu vaccine is showing signs of progress.}

\section{BY JANA SCHLÜTTER}

$\mathrm{W}$ hile the eyes of flu researchers were focused on Asian bird markets in 2009, a pandemic began to emerge at the opposite end of the world in Mexico and California: H1N1 influenza, a version of the 1918 flu virus circulating in pigs for nearly a century, had suddenly leaped back into the human population, which now lacked herd immunity. The virus took the world by surprise.

It took months to develop a vaccine matched to $\mathrm{H} 1 \mathrm{~N} 1$ - too long to thwart the pandemic, which peaked in March 2009 and then again in early November, 2009. "We were fortunate that the virus was not really a nasty bastard and did not kill so many people," says virologist Robert Webster, whose laboratory at St Jude Children's Research Hospital in Memphis, Tennessee, tracks flu viruses and guides the development of flu vaccines to stop them. "If we had an H5N1

virus that was transmitting from human to human: God help us! We would have run out of antivirals almost overnight."

The 2009 outbreak reignited the hunt for a universal vaccine. In contrast to the seasonal flu jab or infection, the spreading pandemic virus, because it was so unlike its predecessors, elicited more cross-reactive, broadly neutralizing antibodies than usual - and now scientists had the technology to find them. One patient even managed to make an antibody that could inactivate all 16 subtypes of the influenza type A virus - the so-called FI6 antibody. (Type A influenza is the most virulent kind, and is responsible for virtually all major human

\section{SNATURE.COM} For some of the latest research on influenza vaccines: go.nature.com/tezyql

\section{flu outbreaks.)}

It was, however, a rare find. Antonio Lanzavecchia, an immunologist at the Institute for Research in Biomedicine in Bellinzona, Switzerland, and his colleagues had to screen 104,000 white blood cells (B cells) collected from eight donors until they found one cell that produced the FI6 antibody. FI6 binds to hemagglutinins representative of all the 16 subtypes of type A influenza, including the $\mathrm{H} 1 \mathrm{~N} 1$ swine flu and avian influenza H5N1, as well as to the more common $\mathrm{H} 3 \mathrm{~N} 2$ strains. In theory, this FI6 antibody provides a blueprint for designing a one-size-fits-all shot. "People have been searching for it forever," says Webster. "Now, it looks as if the Holy Grail for flu really might be achievable."

\section{STEADY TARGET}

The flu virus can skillfully play a chess-like game with the human immune system. Its surface is packed with varieties of two main proteins: neuraminidase and hemagglutinin. Hemagglutinin in particular plays two vital roles: its stem contains the machinery that allows the virus 


\section{TARGETING A SHAPE-SHIFTING VIRUS}

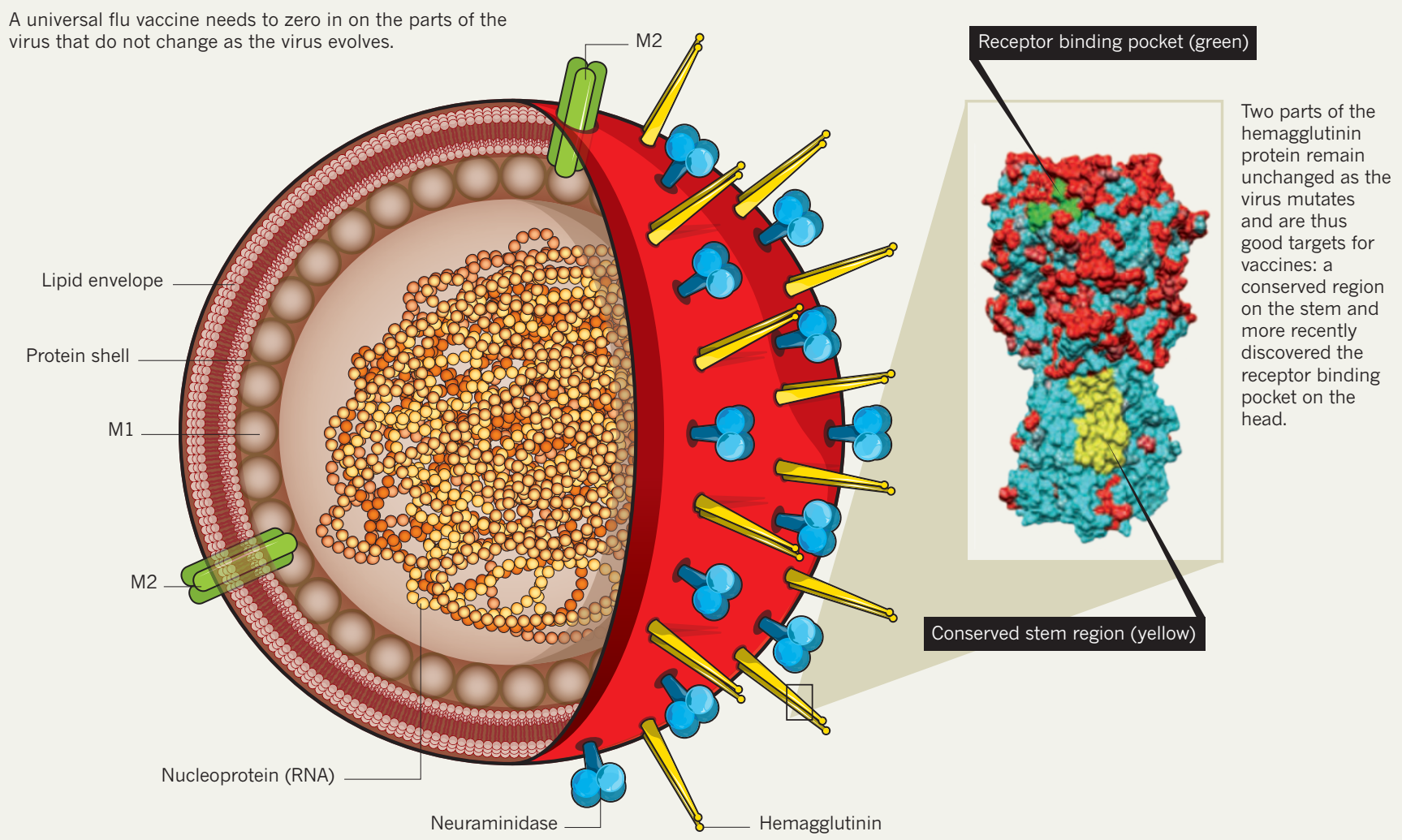

to fuse with host cells; while flashy loops on its globular head act as decoys. To an antibody, each virus appears to be covered with something resembling a dense tropical forest. The freely moving chains of amino acids through the treetops offer alluring binding sites. But these chains mutate and alter their shapes rapidly. The antibody that the host's immune system tailors to fit into the branches of one virus will be limited to that virus, and not work against any new influenza.

Because existing flu vaccines mimic a natural infection, they fall into the same trap: even if our body manages to make a few broadly neutralizing antibodies they are vastly outnumbered by strain-specific ones that bind to the globular head - and most

\section{"When we} understand the basics behind this biology we will be in the driver's seat to design a lot of vaccines." people have to get a flu shot each year to regain immunity.

To sidestep the trap, the immune system would need to refocus its attack, perhaps onto the bases of the trees: a highly stable region of the hemagglutinin stem that tends to stay the same even after the virus mutates. FI6 and most other cross-reactive antibodies push their way in and go for these hard-to-find targets. "This is the first time that an antibody can neutralize every single influenza virus," says Rino Rappuoli, head of vaccines research at Swiss pharmaceutical company Novartis. But while calling FI6 a "milestone", Rappuoli is cautious about seeing it as the key to making a universal vaccine. "That's tough," he says. "We don't know how to do it yet, but it is a dream that can come true."

\section{ROOT OF THE PROBLEM}

There are two ways to use broadly reactive antibodies: as a passive immunization to treat severely ill patients who did not respond to antivirals, or as a template to develop a prophylactic vaccine for everyone. The latter is the more difficult task - it requires sophisticated engineering of the antibody to derive a protein that not only fits the antibody's binding site but also manages to coax the human immune system into producing enough ammunition to prevent infection. Many researchers favour starting with the first option. "It's effective, it's extremely broad, and it's human - there should be no problem injecting antibodies into humans," says Lanzavecchia. "To be frank, our first goal is to use the antibody as a therapeutic."

Gary Nabel, director of the Vaccine Research Center at the National Institute of Allergy and Infectious Diseases (NIAID) in Bethesda, Maryland, agrees on the difficulty in making a universal preventative flu vaccine. "We've been doing similar work with HIV,' he says. Creating a prophylactic vaccine "sounds simple, but it takes time." The challenge, then, is in creating a vaccine that will induce this specific yet unnatural response. Even if one presents a specific site of a protein to the immune system, there are many ways in which antibodies can bind to it. "That's clearly an important problem to solve for any infectious disease, and flu can be our poster child," says Nabel. "When we understand the basics behind this biology we will be in the driver's seat to design a lot of vaccines that could be very, very promising."

While eliciting 'anti-stem' antibodies in humans is difficult, it is not impossible. Nabel and his team were the first to apply a so-called prime-boost approach in humans to achieve this goal. This technique entails giving a combination of two vaccines administered in sequence to induce the strongest immune response possible against a broad array of influenza strains, including H5N1, H5N2 and H9N2. The priming shot was a DNA vaccine: small, circular pieces of bacterial DNA genetically engineered to code for a specific protein that targets an avian flu hemagglutinin. Then, 24 -weeks later, volunteers were given a booster vaccine made of whole inactivated $\mathrm{H} 5 \mathrm{~N} 1$ virus. This regimen enhanced the immune response against avian flu. In addition, three individuals were able to make broadly neutralizing antibodies directed at the hemagglutinin stem, a result that Nabel says is a "proof of concept" for a universal influenza vaccine.

The mice in microbiologist Peter Palese's lab at Mount Sinai School of Medicine in New York are already protected against various flu strains 
by experimental, prophylactic flu vaccines. Palese has shown that two truncated versions of hemagglutinin, one with its globular head chopped off and the other only partly expressed, work better than full-length hemagglutinin, but they're not perfect: they offer good protection against $\mathrm{H} 3$ strains of influenza virus, but they are weaker against H1. Moreover, Palese echoes the familiar caution from anyone working with animal models: "It's promising," he says, "but mice are not men".

Nonetheless, scientists and vaccine companies around the world are hard at work. Advances in high-throughput technology allow them to screen individual B cells from healthy volunteers or patients and search for rare antibodies. In 2008, a group based at the Dutch company Crucell was the first to find broadly neutralizing antibodies against influenza in humans. Apart from exploring therapeutic approaches, they are also trying to vaccinate rodents with conserved viral structures.

Normally, the immune system overlooks the virus's stem (because it's a target that's hard to see and to get to) and produces instead many antibodies that target the viral protein's head. Most broadly neutralizing antibodies, on the other hand, bind to conserved domains on the stem. Crucell is trying to recreate these conserved areas and expose them to the immune system to induce the production of broadly neutralizing antibodies. It's crucial to use the right binding domain, because otherwise the antibody might be just as strain-specific as anything that binds to the head. "We know that we can rebuild the stem in a way that it looks like the binding domain," explains Crucell immunologist Katarina Radosevic. But the question is how to rebuild these structures in a stable form so that they can induce an immune response in animals. "It's a complex task," Radosevic says. "You can't just chop off a part of a molecule and expect the rest to fold and function the same way."

\section{IN THE POCKET}

Recently, another target for a universal vaccine was discovered, one that is conserved but does not hide away in the protein folds. At the crown of hemagglutinin's globular head is the receptor-binding pocket, which allows the virus to infect host cells. With minor modifications, this pocket has the same structure on all the 16 subtypes of hemagglutinin. But the pocket is tiny much smaller than a typical antibody. Nobody expected it to be a good vaccine target, and yet this appears to be the case.

To get snapshots of how the immune system reacts to the seasonal vaccine, researchers from Duke University in Durham, North Carolina, screened antibodies from volunteers several times after vaccination. One of these antibodies, dubbed $\mathrm{CH} 65$, seemed to be a little more effective than others. The Duke team sent the information to Stephen Harrison, a structural biologist at Harvard Medical School and

Children's Hospital in Boston, Massachusetts, for analysis. "We were surprised to find that it docks to the receptor-binding pocket," says Harrison. "About half of the contacts are with amino acid residues in the pocket itself. The degree and intimacy of mimicry is striking."

What's more, point mutations in the area surrounding the pocket did not hinder the antibody from docking. When the researchers tested the antibody against 36 strains of $\mathrm{H} 1$ that had circulated within the last three decades, it blocked 30 of them. "Over 30 years of evolution of the virus, there were hardly any mutations that had a strong effect on binding," Harrison says. Another possibility, he adds, is that since 1977 , the antibody arose so rarely that the virus did not have to escape its attack.

Harrison's research interest is not so much the creation of a universal flu vaccine as in advancing the basic science of affinity maturation: When exposed to the same antigens time and again, the immune system produces antibodies with slight mutations that will bind much more effectively to the invader. But why do some small changes make such a difference to the immune response - and how

"We boost what people already have. We are not trying to prime new responses." can one induce them?

While some scientists are busy mapping the conserved regions on the hemagglutinin molecule, others are still investigating existing vaccine targets that had for many years been the only ones available. These include the ion channel M2, the matrix protein 1 (M1) and the nucleoprotein that the virus needs to keep its genome stable. "We have known about these conserved proteins for at least 20 years," says Rappuoli. While acknowledging that "theoretically" one might prove to be a good target for a universal vaccine, he says that "there hasn't been much progress" on these fronts.

Many companies are still pursuing these targets. VaxInnate, a biotech company in Cranbury, New Jersey, has engineered a hybrid molecule that consists of four copies of M2e - part of the ion channel M2 that sits on the virus surface - fused into the bacterial protein flagellin. VaxInnate recently reported that its vaccine, called Vax102, safely produces an immune response in humans that should be protective against all strains of influenza A. DynaVax, a biotech company in Berkeley, California, has fused M2e and nucleoprotein to create a vaccine candidate called N895; the goal is to encourage antibodies against M2e as well as T cells against the nucleoprotein. Acambis, the UK biotech company based in Cambridge, now owned by Sanofi Pasteur of Lyon, France, evaluated its own M2e vaccine in a phase I study in 2008 and found that it was immunogenic against influenza $\mathrm{A}$, and well-tolerated. The company also conducted another study that it says showed the vaccine protecting about
$70 \%$ of ferrets against bird flu.

Further development has stagnated. Sanofi Pasteur is hesitant about moving its M2e vaccine to phase II trials. "M2e alone is unlikely to be better then the current seasonal vaccines," says Jeffrey Almond, Sanofi's vice president for discovery research and external R\&D. "So we stepped back a little and thought about what we could add to M2e. There is no reason why a universal vaccine shouldn't have multiple components." Meanwhile, Merck, based in New Jersey, has halted its own trials.

\section{A DIFFERENT TACK}

Another path toward a universal vaccine involves not trying to elicit antibodies, but rather boosting the body's T-cell response to infection. While antibodies prevent the virus from infecting host cells, T cells help to clear the virus from the body by killing flu-infected cells. That's the approach being pursued by vaccinologist Sarah Gilbert at the Jenner Institute - a vaccine research organization based at Oxford University in the UK. Gilbert uses an attenuated poxvirus called MVA, which presents the flu nucleoprotein and M1 to the immune system. As a result, Gilbert reports, "we saw a very large peak in T-cell response - everybody improved." This T-cell-mediated immunity might provide another line of defense against flu, possibly in combination with a protein vaccine that targets the stem of the hemagglutinin. Gilbert invited 11 vaccinated volunteers and 11 non-vaccinated volunteers to a quarantine facility. To test their vaccine, the researchers dripped $\mathrm{H} 3 \mathrm{~N} 2$ into the noses of their subjects and monitored their flu symptoms as well as their T-cell response.

Combining Gilbert's vaccine with the seasonal flu shot yields a preparation that boosts not only $\mathrm{T}$ cells but also the antibody response. Following vaccination with the seasonal flu shot alone, young people respond well by producing antibodies, whereas the same vaccine is less effective in older people. "Using both vaccines together might be extremely useful for vaccinating the elderly," Gilbert says. Usually, the seasonal flu jabs don't work as well for older people, because their immune response diminishes quickly, and it becomes more and more difficult to make new antibodies as we age. "We boost what people already have," Gilbert explains. "We are not trying to prime new responses."

Even a potent universal protein vaccine modelled after the perfect antibody would not change the fact that different age groups react differently to a flu jab. Thus a once-in-a-lifetime shot that protect against all strains might never be achieved for everyone: "The question is what universal means," says Rappuoli. "Should it cover all the pandemic strains and the seasonal strains? That's almost impossible. But it's OK to have a dream to move forward."

Jana Schlütter is a science writer in Berlin. 